\title{
PEDESTRIAN GAP ACCEPTANCE BEHAVIOUR IN STREET DESIGNS WITH ELEMENTS OF SHARED SPACE
}

\author{
Ioannis Kaparias \\ Collaborative Transport Hub \\ School of Mathematics, Computer Science and Engineering \\ City University London \\ Northampton Square \\ London EC1V OHB \\ United Kingdom \\ Email: kaparias@city.ac.uk \\ Jignesh Hirani \\ Collaborative Transport Hub \\ School of Mathematics, Computer Science and Engineering \\ City University London \\ Northampton Square \\ London EC1V 0HB \\ United Kingdom \\ Email: jignesh.hirani.1@ city.ac.uk
}

Michael G.H. Bell

Institute of Transport and Logistics Studies

University of Sydney

St James Campus C13

173-175 Phillip Street

Sydney NSW 2000,

Australia

Email: michael.bell@sydney.edu.au

\author{
Bill Mount \\ Centre for Transport Studies \\ Department of Civil and Environmental Engineering \\ Skempton Building, South Kensington Campus, \\ Imperial College London, \\ London SW7 2BU \\ United Kingdom \\ Email: bill.mount49@googlemail.com
}

$(6,184$ words $+3 \cdot$ Figures $(250)+2 \cdot$ Tables $(250)=7,434$ words +37 references $)$

This paper was submitted to the Transportation Research Board.

2 March 2016 


\begin{abstract}
Recent developments in the field of urban street design have seen the emergence of the concept of "shared space", which is a term that collectively refers to a range of streetscape treatments aiming at creating a better public realm by asserting the function of streets as places and designing more to a scale aimed at easier pedestrian movement and lower vehicle speeds. In light of this shift in focus towards the pedestrian, this paper examines the aspect of pedestrian gap acceptance behaviour and how this may change as a result of the implementation of street layouts with elements of shared space. Using video data from London's Exhibition Road site during periods before and after its conversion from a conventional dual carriageway to a layout featuring a number of elements of shared space, the study looks at changes in terms of key gap acceptance variables, such as waiting time, crossing time, crossing speed and critical gap. The effects of a number of traffic- and pedestrian-specific attributes on gap acceptance are also investigated by means of binary logistic regression modelling. The results suggest that pedestrians feel more comfortable and confident in their interaction with vehicles postredevelopment of the site, as not only do they tend to accept shorter gaps in traffic, but they also appear to be more at ease when crossing. In particular elderly and pedestrians travelling with children seem to benefit the most, as they no longer appear to be any less comfortable when crossing the road than other pedestrians.
\end{abstract}




\section{$1 \quad$ INTRODUCTION}

Urban street design has traditionally been very closely tied with road safety. The latter has been a concern since the introduction of motorised vehicles, and became paramount with mass motorisation from the 1950s onwards. Of particular importance was the protection of pedestrians, who, being more vulnerable, faced greater risk of suffering injury or death. This was pursued by means of their segregation from vehicular traffic, which, dating back at least to the work of Le Corbusier in the 1930s, relied upon the design and implementation of structures including pedestrian subways and bridges, as well as guardrails and walls separating pedestrian pathways from the road, which in turn was reserved for vehicles. The concept is set out most lucidly in Buchanan's 'Traffic in Towns" report (1) of 1963, which served as a street design manual in the UK for many decades.

In recent years, however, there has been a trend away from traffic segregation, driven by developments in architecture and urban planning. Instead, street design has shifted gradually towards the concept of "shared space" as a means of creating a better public realm, mainly by asserting the function of streets as places rather than arteries and designing more to a scale aimed at easier pedestrian movement and lower vehicle speeds. Elaborating more on the term "shared space", and conversely to popular belief, this is not used to characterise entire streets and places as "shared" or "not shared", particularly given that streetscape design cannot be standardised and needs to be context-sensitive. Instead, shared space is used as an "umbrella" term to collectively refer to a range of streetscape treatments, aiming at creating a more pedestrian-friendly environment. These may range from the removal of guardrails and the introduction of "informal" (uncontrolled) pedestrian crossing facilities in a traditional "kerbed" street layout, through to radically-engineered layouts with a single surface and little or no delineation between pedestrian and vehicle areas (2-5). Recent guidance published by the UK Department for Transport (DfT) defines shared space as "a street or place designed to improve pedestrian movement and comfort by reducing the dominance of motor vehicles and enabling all users to share the space rather than follow the clearly defined rules implied by more conventional designs" (6). Examples of streets with varying extents of shared space elements can be found around the world and include: the concept of "woonerf" and "home zone" in residential areas in the Netherlands and UK respectively; the "Manual for Streets" approach in the UK (7-8); and the "Complete Streets" initiative in the USA (9).

As identified by the UK DfT guidance, pedestrian comfort is central to the successful operation of street designs with elements of shared space. Through such designs, it is aspired to allow pedestrians to "colonise" the street, and this can only be achieved if they feel confident enough to move around freely and interact with vehicles. It is, hence, important to investigate the issue of pedestrian comfort in shared space, and previous work by the authors has already gone some way in this direction by looking at traffic conflicts (10-11), behavioural interactions (12), as well as perceptions of pedestrians (13).

The aim of the present study is to extend the knowledge gathered so far on pedestrian comfort in shared space by investigating pedestrian gap acceptance behaviour, and specifically how this may change as a result of the implementation of street layouts with elements of shared space. Indeed, gap acceptance is often used as a proxy for the comfort and confidence of road users (14), and the results are, hence, expected to complement previous findings effectively. The analysis is conducted by means of video observations from the Exhibition Road site in London's South Kensington area during periods before and after its conversion from a conventional dual carriageway to a layout featuring a number of elements of shared space.

The present paper is structured as follows: Section 2 presents the background of the study, focussing primarily on previous research on the topic of pedestrian gap acceptance. Section 3 then goes on to present the study site, the data collection method employed, and the analysis methodology. Section 4 presents the results of the analysis, and discusses the trends in pedestrian gap acceptance behaviour in relation to elements of shared space. Section 5, finally, concludes the paper and identifies areas of future research. 


\section{BACKGROUND}

A gap is defined in the Highway Capacity Manual (HCM 2010) as "the elapsed time interval (time headway) between arrivals of two successive vehicles in the major stream at the same reference point" (15). In the frequently encountered traffic situation where a minor traffic stream joins or crosses a major one (e.g. a T-junction, four-arm intersection or roundabout), drivers in the former seek for adequately long gaps in the latter to merge or cross, and hence assess the times between oncoming vehicles (gaps) in the major stream. This is called gap acceptance behaviour (in what a gap is accepted if the driver merges or crosses, but rejected otherwise), and many studies have concentrated on its monitoring with a view of drawing conclusions on various behavioural aspects of traffic.

Gap acceptance analysis studies are generally concerned with two aspects: establishing the value of the so-called "critical gap", and developing behavioural models to describe gap acceptance behaviour. As concerns the former aspect, the critical gap is defined as the minimum accepted gap allowing the entry of a vehicle in the major stream. This corresponds to the gap accepted by $50 \%$ of the drivers (Greenshields's definition (16)), or to the gap for which the number of accepted gaps shorter than it is equal to the number of rejected gaps longer than it (Raff's definition (17)), with several other definitions having been provided in the literature (18). With regard to the latter aspect, several behavioural models expressing the probability of accepting a gap in traffic under different conditions have been developed on the basis of real-world observations and measurements, but also simulation models, and it has been found that a wide range of driver-specific and environment-specific factors influence gap acceptance behaviour, as, for example, in (19).

But while gap acceptance monitoring was initially conceived as a method of modelling traffic encounters between vehicle streams, it has been also extensively used to describe pedestrian crossing behaviour, where pedestrians seek an adequately long vehicular gap to cross the road, and accept or reject available gaps accordingly (20-22). The vehicular gap is defined as the time difference between two oncoming vehicles with reference to the pedestrian path, and it has been found that pedestrians exhibit a similar behaviour to drivers, in the sense that they too have a critical vehicular gap which determines their crossing behaviour. In fact, a large number of studies from different countries have concentrated on the determination of the pedestrian critical gap, with results varying from about 3 seconds to up to 10 seconds (23). In addition to the critical gap, pedestrian crossing behaviour has also been looked at from the point of view of pedestrian speed (24). Specifically, it was shown by one of the first studies on the topic (25) that there is a link between pedestrian crossing speed and gap acceptance, in what pedestrians accepting shorter gaps tend to cross faster. Further research has concentrated on how the pedestrian speed changes during a crossing (26), and how its value varies between compliant and non-compliant crossing behaviour (27).

Other research has focused on the factors affecting pedestrian crossing behaviour and has devised models to describe this. Overall, pedestrian crossing behaviour has been found to depend on a number of factors, such as: the type and speed of the vehicle, and the waiting time of the pedestrian (28); the traffic volume and the weather and visibility conditions (29); the width of the road (30); the existence of a so-called "rolling gap", which reflects the situation when a pedestrian needs to cross multiple lanes of traffic (31); the layout of the junction/crossing and its implication on the perceived safety of the pedestrian (32); the presence of parked vehicles (33); and the prevalence of non-compliant crossing behaviour by other pedestrians (34). Furthermore, several studies have investigated pedestrian gap acceptance behaviour in relation to pedestrian-specific characteristics. For instance, it has been found that females tend to wait longer than males when attempting to cross the road (35), and that children and elderly tend to be more cautious and hesitant compared to young and middle-aged adults, which results in the acceptance of longer vehicular gaps (22).

A small number of studies have also looked at the specific case of pedestrian crossing behaviour in street environments with elements of shared space. Most notably, in previous work by the authors a qualitative framework to model behavioural pedestrian-vehicle interactions was developed, and through 
its application in a recently redeveloped urban road site, it was found that pedestrians demonstrate increased confidence in their interaction with vehicles, while the behaviour of drivers remains unchanged (12). The study, however, focused mostly on qualitative behavioural characteristics and did not measure gap acceptance. In another study by Clarkson et al (36), pedestrian gap acceptance was measured in three different shared space sites in the UK, alongside driver yielding behaviour, and it was found that the higher numbers of pedestrians found in shared space layouts and their greater assertiveness have a positive impact on vehicle yielding behaviour, which in turn affects pedestrian comfort, as expressed by gap acceptance behaviour. However, the study only looked at redeveloped shared space sites and did not draw any comparisons with the pre-redevelopment situation.

The present study extends existing knowledge by investigating pedestrian gap acceptance behaviour through video observation of a street site before and after its redevelopment as a layout with elements of shared space, and the next section outlines how this is done.

\section{METHODOLOGY}

The data collection and analysis methodology are outlined in this section. This includes a description of the site (Exhibition Road, South Kensington, London), and is followed by an account of the data collection methods and tools employed, and of an explanation of the analysis method used.

\subsection{Site description}

Exhibition Road is an $800 \mathrm{~m}$ long road located in the Royal Borough of Kensington and Chelsea (RBKC) in London and is home to a number of London's most popular museums (Natural History, Science, V\&A). The surrounding area of South Kensington is well-known as a cultural centre, including other venues such as the Royal Albert Hall as well as many academic institutions, including Imperial College London. As the previous conventional dual carriageway layout of Exhibition Road was crowded (a problem exacerbated by numerous pedestrian barriers) and dominated by high traffic flows and parked vehicles, the RBKC undertook an engineering scheme, the "Exhibition Road Project", which included its redevelopment featuring a number of elements of shared space (Figure 1).

\section{[Figure 1 here]}

The project was implemented over four years from mid-2008 to completion in late 2011. More specifically, the following three main streetscape treatments were carried out:

1. Re-allocation of street space (Figure 1a): The previous layout of the $24 \mathrm{~m}$ wide Exhibition Road consisted of a $16 \mathrm{~m}$ wide dual carriageway, accommodating one lane of traffic in each direction as well as excess width allocated to parked vehicles, and of two $4 \mathrm{~m}$ wide footpaths on either side of the carriageway, accommodating pedestrians. As a result of the redevelopment, traffic was shifted to the eastern side of the road to occupy a single carriageway of $8 \mathrm{~m}$ width (termed the "traffic zone"), with the former western side of the dual carriageway becoming a so-called "transition zone", accommodating primarily pedestrians, but also parking, cycles and coaches alighting to drop-off or pick-up passengers. The two $4 \mathrm{~m}$ footpaths remained in place and formed the so-called "pedestrian zone". The space also saw the removal of the kerbs and the implementation of an end-to-end single surface with an $800 \mathrm{~mm}$ corduroy tactile delineator to indicate the start of the pedestrian zone on each side of the road.

2. Unravelling of a one-way system (Figure $1 \mathrm{~b}$ and $1 \mathrm{c}$ ): In the original layout, a one-way system was in place around the South Kensington Station area, whereby the southbound traffic was led along the southern tip of Exhibition Road and along Thurloe Street, while the northbound traffic was guided along Thurloe Place. As a result of the redevelopment, Thurloe Place was converted 
to a two-way street, accommodating both the northbound and the southbound traffic, while Thurloe Street was converted to an access-only street.

3. Re-design of pedestrian crossing facilities (Figure 1d): At the intersection of Exhibition Road with Cromwell Road, the original design included a staggered north-south pedestrian crossing on the western side of the site, which, however was not following the desire-lines and required pedestrians to cross in two stages, thus resulting in a high number of jaywalkers. The redevelopment removed the staggered crossing and replaced it with a wide $(12 \mathrm{~m})$ straightacross crossing, allowing pedestrians to complete their crossing in a single phase. The scheme also included the removal of pedestrian guardrails and other street clutter to further facilitate pedestrian movement. Some vehicle movements were also prohibited.

\subsection{Data collection}

Video footage has been collected through high-mast cameras for periods before and after the implementation of the Exhibition Road scheme as part of recent studies analysing traffic conflicts and behavioural interactions in the area (10-12). This has also been complemented by vehicle traffic and pedestrian crossing counts, in order to relate to changes in the traffic conditions around the site. In this study, the data collected is used to assess the impact of the new design of Exhibition Road on pedestrian gap acceptance. In the before-case, the data refers to August 2008, prior to the start of the redevelopment works, and has been collected from a number of critical locations in terms of pedestrian crossing occurrences, as identified previously (10). For the after-situation, the video footage comes from the same locations for periods between October and December 2011, following the completion of the scheme.

[Figure 2 here]

The locations are the following (Figure 2):

- L1: Exhibition Road main body (Before: Cameras A \& B - After: Cameras 4, 5, 6 \& 7): In the original layout, pedestrians wishing to cross Exhibition Road at this location (entrances of V\&A, Natural History and Science museums) needed to detour by more than $100 \mathrm{~m}$ to reach the closest formal pedestrian crossing; as a result, they chose to cross freely. The new layout facilitates those crossing movements (Figure 1a).

- L2: Cromwell Road junction (Before: Cameras C \& D - After: Cameras E, F, G \& H):

In the original layout, the facilities provided to pedestrians wishing to cross Cromwell Road to continue walking on either the eastern or the western kerbsides of Exhibition Road were two staggered pelican crossings, which required a detour and often long waiting times for a green man signal. As a result, the vast majority of the pedestrians used "shortcuts" bypassing the staggered crossings and jaywalking, thus coming into conflict with right-turning southbound traffic from Exhibition Road in the case of the western crossing, or with left-turning southbound traffic in the case of the eastern crossing. The western crossing has been replaced by a wide straight-across crossing in the new layout, while the staggered crossing on the eastern side has been retained but redesigned (Figure 1d).

- L3: Thurloe Street (Before: Camera F - After: Cameras 1 \& 2):

Pedestrians using this location in the original layout were faced with two problems: the lack of pedestrian crossing facilities, and the insufficient space for pedestrians on the southern kerbside of the road, such that footpath overcrowding resulted in a large number of free crossings. In the new layout, this location has been redesigned as "access-only", giving more space to pedestrians (Figure 1b). 


\subsection{Data processing and analysis}

As analysing the complete duration of the video data would take up a significant amount of time and provided that peak and off-peak periods exist in pedestrian crossing levels as a result of peak and offpeak traffic and pedestrian flows, five hours of analysis for each location for the before- and after-case are selected, with a mix of week and weekend days chosen depending on the location and the availability of video data. These are:

- Weekdays: 08:00 - 09:00 (morning rush hour, offering an insight of the local residents' and workers' use of the road), 12:00 - 13:00 (midday, when a large number of tourists enter and exit the museums) and 17:00 - 18:00 (evening rush hour, with tourists and workers leaving the area, and locals returning)

- Weekends: 12:00 - 13:00 and 17:00 - 18:00 (again midday and evening rush hour).

Hence, a total of 30 hours of video footage are processed with respect to pedestrian gap acceptance occurrences. As, however, Thurloe Street (L3) has become an "access-only" street post-redevelopment, no pedestrian gap acceptance events occur any longer, and so the respective five hours of video are omitted, bringing the total number of hours analysed to 25 (L1 before and after, L2 before and after, L3 before only).

The processing involves, firstly, identifying pedestrian gap acceptance/rejection occurrences in the videos. This is done "manually" according to the behavioural analysis framework defined in (12), whereby only "Steady Car - Pedestrian (SC-P)" interaction events are considered as such occurrences. These are defined as follows: "The vehicle involved in the interaction is a four-wheeler (i.e. car, van etc. - generalised as 'Car' for convenience) and is already travelling at a steady pace at the time of interaction with a pedestrian. This means that the vehicle's movement is perceived to be independent rather than a result of a reaction to a previous interaction event" (12). As such, only those instances where a pedestrian (or a group of pedestrians) is making a decision to (not) cross the vehicle zone or carriageway freely under the presence of an oncoming vehicle are classified as gap acceptance/rejection occurrences; this includes occurrences of crossing on a red man signal, which are legal in the UK. It should be made clear, though, that not all pedestrian crossing events are counted as gap acceptances or rejections; for instance, occurrences when pedestrians cross the carriageway and no vehicles are present, or instances when pedestrians cross at a signalised crossing with stopped vehicles at a red light (corresponding to so-called "Effective Shared Space (ESS)" interaction events in (12)), are discarded.

After the identification of a pedestrian gap acceptance/rejection event, the next step of the processing involves the measurement of a number of key variables, namely: gap length (in seconds) and vehicle speed (in $\mathrm{km} / \mathrm{h}$ ), and in case of acceptance, pedestrian crossing time (in seconds), crossing speed (in $\mathrm{km} / \mathrm{h}$ ) and total waiting time (in seconds). Furthermore, in addition to the crossing-specific variables, some visible pedestrian-specific characteristics are also recorded. Given that the video has been collected from high-mast cameras, however, such characteristics include only the ones relating to the number and type of pedestrians, so, for instance, whether the pedestrian is travelling alone or in a group, and whether elderly and/or children are present.

The analysis of the collected data then proceeds in two stages. In the first stage general statistics of the various variables describing pedestrian gap acceptance behaviour are presented for each location observed, including the calculation of the value of the critical gap before and after the site redevelopment using Raff's method (17). This allows for a comparison of pedestrian crossing behaviour in the different locations of the site in relation to elements that have changed as a result of the redevelopment. In the second stage, then, an insight into the causes of these changes is given through the derivation of binary logistic regression models, as defined in (37), describing the pedestrian gap acceptance behaviour around the site as a function of certain relevant factors. 


\section{$4 \quad$ RESULTS}

The video data collected are processed and analysed using the method described in Section 3.3, and the results are presented in this section.

\subsection{Gap acceptance behaviour trends}

The key variables describing gap acceptance behaviour around the Exhibition Road site before and after redevelopment to a design with elements of shared space are shown in Table 1, in which part (a) presents the measures related to the prevailing traffic conditions, and part (b) shows the ones related to the pedestrian crossing behaviour.

[Table 1 here]

Looking at Table 1a, the average gap length encountered by pedestrians appears to be slightly lower post-redevelopment at both the main body of Exhibition Road (L1) and at the Cromwell Road junction (L2), though the results of corresponding T-tests indicate that these differences are not statistically significant at the 0.05 level ( $p$-values of 0.299 and 0.097 respectively mean that the null hypotheses that the before and after values at both locations come from the same populations cannot be rejected). As such, it can be concluded that pedestrians are faced with similar gap distributions when attempting to cross both pre- and post-redevelopment, as well as on Thurloe Street (L3), whose gap distribution is similar. What appears to have changed, however, is the average vehicle speed, which is slightly lower at the Cromwell Road junction (L2) and substantially lower at the main body of Exhibition Road (L1) after the redevelopment. T-tests confirm that these differences are statistically significant at the 0.05 level ( $p$-values of 0.000 for both locations suggest that the null hypotheses that the values come from the same distributions are rejected), which means that indeed pedestrians are faced with similarly dense but slower traffic after the redevelopment at L1 and L2. No significant differences are reported with respect to the variability of either gap length or vehicle speed around the site before or after the redevelopment.

Looking at the pedestrian crossing behaviour as illustrated by the measurements in Table 1b, it can be clearly seen that the waiting time, crossing time and crossing speed across all three locations of the site are of a similar order before redevelopment, with the notable exception of the waiting time at the Cromwell Road junction (L2), which is higher. This is expected, as vehicle traffic is heavier at that location, and pedestrians (crossing freely on a red man signal in the staggered crossing layout) usually have to wait longer for an acceptable gap. A further feature of interest is the greater variability of the waiting time, crossing time and crossing speed at the main body of Exhibition Road (L1) before redevelopment (expressed by the corresponding higher values of the coefficient of variation, i.e. the ratio of the standard deviation to the mean), which could be an indication of the area's unmet need to function as a place where pedestrians go to spend time, in addition to its intended function as an artery which pedestrians need to cross. Indeed, pedestrians whose needs are not met by the layout's function may end up waiting much longer or much shorter than average, and may cross much faster or slower than average, which would result in greater variability in the waiting time, crossing time and crossing speed values encountered; on the other hand, if the layout meets the needs of all pedestrians, a more uniform crossing behaviour is to be expected, with correspondingly smaller variability in the values measured.

Considering the crossing behaviour of pedestrians after the redevelopment, some very interesting findings are obtained. The first notable result is the significant reduction in the average pedestrian waiting time at the Cromwell Road junction (L2), which is coupled with a slight increase in the average crossing time and a slight decrease in the average crossing speed. These are all found to be statistically significant at the 0.05 level ( $p$-values of $0.000,0.004$ and 0.006 respectively, meaning that the null hypotheses that they are equal to the before-case cannot be rejected), and may be an indication 
of greater pedestrian comfort and confidence provided by the new more "open" layout, and particularly the absence of guardrails and other street clutter, despite the only marginal reduction of average vehicle speed. This is a finding that is in line with the results of the qualitative behavioural analysis study conducted previously (12).

A further noteworthy result is the slight increase of the mean crossing time and the slight decrease of the mean crossing speed at the main body of Exhibition Road (L1), both of which are statistically significant at the 0.05 level ( $p$-values of 0.021 and 0.017 respectively, meaning that the null hypotheses that they are the same as in the before-case are rejected), and which, again, may suggest increased comfort among pedestrians when crossing the traffic zone. This is also supported by the lower variability of crossing times and speeds at this location (expressed by the lower coefficient of variation values), which may be indicating that the new layout is responding more appropriately to pedestrian needs, catering a stronger sense of place (hence pedestrian crossing behaviour is more uniform). There is also a marginal increase in the mean waiting time, though this is found to be statistically insignificant at the 0.05 level ( $p$-value of 0.820 , which means that the null hypothesis that there is no change cannot be rejected).

The last variable investigated is the critical gap. This is established using Raff's method (16), which involves plotting the cumulative distribution curves for accepted and rejected gaps and finding the point of their intersection, which corresponds to the gap length that is accepted by as many pedestrians as it is rejected by. The cumulative distribution plots of accepted and rejected gaps at each location and time (before or after) in the Exhibition Road site are shown in Figure 3. As can be seen from the left hand side of the figure, the critical gap in all three locations before the redevelopment has a similar value, and specifically it is 5.2, 5.1 and 4.9 seconds for the Exhibition Road main body (L1), the Cromwell Road junction (L2) and Thurloe Street (L3) respectively. After the redevelopment, however, this appears to drop significantly, and specifically to 4.85 seconds at the Cromwell Road junction (L2) and to 4.45 seconds at the main body of Exhibition Road (L1). This result further supports the finding of increased pedestrian comfort and confidence around the space as a result of the redevelopment and the associated impacts on vehicle traffic (lower flow and speed).

[Figure 3 here]

\subsection{Gap acceptance behaviour modelling}

In order to determine how pedestrian gap acceptance is influenced by different factors before and after the redevelopment of Exhibition Road to a design with elements of shared space, binary logistic regression is performed, as this seems to be most suited due to the fact that gap acceptance is a phenomenon with a binary outcome (Rejection $=0$, Acceptance $=1$ ). Different regression models are fit for each of the three locations of the Exhibition Road site, before and after redevelopment (only before for L3), the outcome of which is the probability of a pedestrian to accept a gap in traffic and cross the traffic zone or carriageway.

In the resulting models, gap acceptance is the dependent variable, which is to be predicted by five independent variables: travel as a group, presence of elderly, presence of children, gap length, and vehicle speed. In order for the resulting model coefficients to be comparable with each other, however, the independent variables need to be binary, and while the former three are already such (denoted "Group", "Elderly" and "Children") and have only yes-no levels (taking 0-1 values), the latter two are continuous and need to be converted to binary. As such, the binary variables "Long gap" and "Fast vehicle" are created, which correspond to the high gap length and vehicle speed values located beyond one standard deviation of the mean, as outlined in Table 1 (which, assuming normal distribution, corresponds to the upper 32.8-th percentile).

The SPSS 22 statistical software package is used to perform binary logistic regression and estimate the coefficients of the resulting logit models for pedestrian gap acceptance. Five models are estimated, and the results are shown in Table 2. Looking at the models for the before-case first, shown 
in the left column of Table 2, it can be seen that all models for the three locations have significant negative constants (at the 0.05 level), which denote that the starting position for a pedestrian assessing a gap when all other attributes are at their zero levels is on rejection. This is a sensible finding, as pedestrians are unlikely to automatically feel comfortable to cross the vehicle zone or carriageway.

[Table 2 here]

As concerns the other attributes, gap length appears to have a significant positive effect at the 0.05 level on gap acceptance at all three locations, while vehicle speed seems to have a negative significant effect in two of the three models. This is also sensible, as longer gaps and lower speeds are likely to give greater confidence to pedestrians to cross. Considering the locations, it appears that these effects are more dominant at the Cromwell Road junction (L2), rather than at the main body of Exhibition Road (L1) and on Thurloe Street (L3); a reason could be the fact that the staggered pelican crossing across a four-lane highway layout implies that vehicles have absolute priority at that location, which makes pedestrians behave more "conservatively" and seek even longer gaps and even lower vehicle speeds. Furthermore, significant negative effects of "Elderly" and "Group" on gap acceptance are reported at the Cromwell Road junction (L2), which indicates the smaller likelihood of a gap being accepted for elderly pedestrians and for pedestrians travelling as a group. Similarly, significant negative effects are reported for the "Elderly" and "Children" attributes at Thurloe Street (L3).

Looking at the two models for the after-case in the right column of Table 2, again it can be seen that they have significant negative constants at the 0.05 level, slightly lower than their before-case counterparts. This means that, again, the starting position for a crossing pedestrian is to reject a gap, though this is slightly weaker than in the before-case. Regarding the other attributes, and looking at the Exhibition Road main body (L1), it can be seen that only "Long gap" has a positive significant effect on gap acceptance, which is lower than the corresponding effect in the before-case. This suggests that pedestrians are now more likely to accept shorter gaps than before, and it is noteworthy that the previous significant effect of vehicle speed is not significant any longer in the after-situation. The result, hence, confirms the finding of greater confidence at this location post-redevelopment, as established in the first stage of the analysis.

A similar trend is observed at the Cromwell Road junction (L2), for which the corresponding after-model shows, again, a significant negative constant, and significant positive and negative effects for gap length and vehicle speed respectively. These are, again, lower than their counterparts in the before-model, and, hence, reinforce the finding of increased confidence of crossing pedestrians. An interesting feature is that the previously significant negative effects for "Group" and "Elderly" are not significant anymore at the 0.05 level, which suggests that elderly pedestrians and pedestrians in groups now have greater confidence when crossing. It should, however, be also noted, that these coefficients remain lower than the corresponding Exhibition Road main body (L1) ones, which, as expected, shows that pedestrian confidence in crossing the vehicle zone remains higher at the latter location. This is most likely due to the implementation of the single surface and the shared space features facilitating free pedestrian crossings at L1, combined with the lower vehicle traffic flows and speeds, as opposed to the more pedestrian-friendly, yet still conventional layout at L2.

\section{CONCLUSIONS}

In light of the shift in focus in urban street design, this paper has examined the topic of pedestrian gap acceptance behaviour and how this changes as a result of the implementation of street layouts with elements of shared space. Using video data from the Exhibition Road site in London during periods before and after its conversion from a conventional dual carriageway to a layout featuring a number of elements of shared space, changes in terms of key gap acceptance variables have been investigated, such as waiting time, crossing time, crossing speed and critical gap. The effects of a number of traffic- 
and pedestrian-specific attributes on gap acceptance have also been investigated by means of binary logistic regression.

The results suggest that pedestrians show greater comfort and confidence in their interaction with vehicles post-redevelopment of the site, as not only do they tend to accept shorter gaps in traffic, but they also appear to be more at ease when crossing, walking slower and taking more time. The reduction in the average waiting time and vehicle speed in certain cases, as brought about by the new layout, does, naturally, play an important role. In particular vulnerable road user groups, such as elderly and pedestrians travelling with children, seem to benefit the most, as they no longer appear to be any less comfortable when crossing the road than other pedestrians.

Naturally, the findings of this study have implications on policy and practice as concerns the planning and design of successful street design schemes with elements of shared space. For example, the finding of greater confidence for crossing pedestrians following the implementation of the scheme, and in particular the increased comfort of vulnerable pedestrians, is instrumental for policy-makers, who wish to conduct an a priori impact assessment of a proposed scheme. Coupled with traffic conflicts analysis, this finding may act as a surrogate safety analysis method, highlighting potential hazards in advance of the implementation, and enabling planners to take them on board at the early stages of the design. Alternatively, combined with interviews and further revealed preference surveys, the results could facilitate an a priori evaluation of the projected acceptance of a scheme by different road user groups. Also, by extending the study to different sites and settings, these results could form the beginnings of the calibration stage of an advanced microsimulation model, which would take into account the behaviour of the various road users and would predict their trajectories, thus facilitating the work of practitioners.

While this study has thrown some light into the topic of pedestrian gap acceptance in street layouts with elements of shared space, there are several future research directions that remain to be explored next. For instance, a limitation of the present study has been the fact that observations and measurements have been conducted manually; by employing image processing algorithms in conjunction with gap acceptance monitoring as a next step, it will be possible to automate the process and conduct observations across different sites for longer periods. This will additionally enable the collection of more detailed data for the analysis of additional elements, such as the impact of the changes in the traffic patterns on gap acceptance. Furthermore, future work could concentrate on monitoring the gap acceptance of other road users in a wide range of shared space environments, such as drivers and cyclists, which would deliver much different conclusions and models. In particular, it would be useful to analyse the gap acceptance behaviour of road users with disabilities (e.g. blind and partially-sighted, hearing impaired and people with learning difficulty) in more detail, especially given that a certain discomfort towards the concept of shared space has often been reported by a number of disability groups. Finally, the results obtained could be complemented by road user surveys, from which it will be possible to more systematically investigate the views and perceptions of pedestrians with respect to their gap acceptance behaviour.

\section{ACKNOWLEDGEMENT}

The authors would like to thank the Royal Borough of Kensington and Chelsea for supporting this work by providing data and guidance.

\section{REFERENCES}

(1) Buchanan C, Cooper GHC, MacEwen A, Crompton DH, Crow G, Michell G et al. Traffic in towns, HMSO, 1963. 
(2) Hamilton-Baillie, B. Urban design: Why don't we do it in the road. Journal of Urban Technology, Vol. 11, 2004, pp. 43-62.

(3) Hamilton-Baillie, B and Jones, P. Improving traffic behaviour and safety through urban design. Proceedings of the Institution of Civil Engineers - Civil Engineering, Vol. 158, 2005, pp. 39-47.

(4) Hamilton-Baillie, B. Towards shared space. Urban Design International, Vol. 13, 2008, pp. 130138.

(5) Hamilton-Baillie, B. Shared space: Reconciling people, places and traffic. Built Environment, Vol. 34, 2008, pp. 161-181.

(6) UK Department for Transport. Local Transport Note 1/11 - Shared space, 2011.

(7) UK Department for Transport. Manual for Streets. 2007.

(8) Chartered Institute of Highways and Transport. Manual for Streets 2 - Wider application of the principles. 2010.

(9) LaPlante, JN and McCann, B. Complete streets in the United States, $90^{\text {th }}$ Annual Meeting of the Transportation Research Board, Washington, DC, USA, 2011.

(10) Kaparias, I, Bell, MGH, Greensted, J, Cheng, S, Miri, A, Taylor, C and Mount, B. Development and implementation of a vehicle-pedestrian conflicts analysis method: Adaptation of a vehiclevehicle technique. Transportation Research Record, Vol. 2198, 2010, pp. 75-82.

(11) Kaparias, I, Bell, MGH, Dong, W, Sastrawinata, A, Singh, A, Wang, X and Mount, B. Analysis of pedestrian-vehicle traffic conflicts in street designs with elements of shared space. Transportation Research Record, Vol. 2393, 2013, pp. 21-30.

(12) Kaparias, I, Bell, MGH., Biagioli, T, Bellezza, L and Mount, B. Behavioural analysis of interactions between pedestrians and vehicles in street designs with elements of shared space, Transportation Research Part F: Traffic Psychology and Behaviour, Vol. 30, 2015, pp. 115-127.

(13) Kaparias, I, Bell, MGH, Miri, A, Chan, C and Mount, B. Analysing the perceptions of pedestrians and drivers to shared space, Transportation Research Part F: Traffic Psychology and Behaviour, Vol. 15, 2012, pp. 297-310.

(14) Findley, D.J., Schroeder, B.J., Cunningham, C.M. and Brown, T. Highway Engineering: Planning, Design and Operations. 2015.

(15) Transportation Research Board. Highway Capacity Manual. 2010.

(16) Greenshields, BD, Shapiro, D and Ericksen, EL. Traffic performance at urban street intersections. Technical Report 1, Bureau of Highway Traffic, Yale University, New Haven, CT, 1946.

(17) Raff, MS and Hart JW. A volume warrant for urban stop signs. Eno Foundation for Highway Traffic Control, 1950.

(18) Brilon, W, Koenig, R and Troutbeck, RJ. Useful estimation procedures for critical gaps. Transportation Research Part A: Policy and Practice, Vol. 33, 1999, pp. 161-186.

(19) Daganzo, CF. Estimation of gap acceptance parameters within and across the population from direct roadside observation. Transportation Research Part B: Methodological, Vol. 15, 1981, pp. $1-15$.

(20) DiPietro, CM and King, LE. Pedestrian gap-acceptance. Highway Research Record, Vol. 308, 1970, pp. 80-91.

(21) Himanen, V and Kulmala, R. An application of logit models in analyzing the behavior of pedestrians and car drivers on pedestrian crossings. Accident Analysis \& Prevention, Vol. 20, 1988, pp. 187-197.

(22) Oxley, J, Ihsen, E, Fildes, BN, Charlton, JL and Day, RH. Crossing roads safely: An experimental study of age differences in gap selection by pedestrians. Accident Analysis \& Prevention, Vol. 37, 2005, 962-971.

(23) Chandra, S, Rastogi, R and Das, VR. Descriptive and parametric analysis of pedestrian gap acceptance in mixed traffic conditions. KSCE Journal of Civil Engineering, Vol. 18, 2014, pp. 284-293.

(24) Rastogi, R, Chandra, S, Vamsheedhar, J and Das, VR. Parametric study of pedestrian speeds at midblock crossings. Journal of Urban Planning and Development, Vol. 137, 2011, pp. 381-389. 
(25) Moore, RL. Pedestrian choice and judgment. Journal of the Operational Research Society, Vol. 4, 1953, pp. 3-10.

(26) Kadali, BR and Vedagiri, P. Marked versus unmarked pedestrian road crossing behavior at uncontrolled midblock crosswalk in mixed traffic condition. $92^{\text {nd }}$ Annual Meeting of the Transportation Research Board, Washington, DC, USA, 2013.

(27) Cherry, C, Donlon, B, Yan, X, Moore, SE and Xiong, J. Illegal mid-block pedestrian crossings in China: gap acceptance, conflict and crossing path analysis. International Journal of Injury Control and Safety Promotion, Vol. 19, 2012.

(28) Sun, D, Ukkusuri, SVSK, Benekohal, RF and Waller, ST. Modeling of motorist-pedestrian interaction at uncontrolled mid-block crosswalks. $82^{\text {nd }}$ Annual Meeting of the Transportation Research Board, Washington, DC, USA, 2003.

(29) Andrew, HW. Factors influencing pedestrian cautiousness in crossing streets. Journal of Social Psychology, Vol. 131, 1991, pp. 367-372.

(30) Cohen, J, Dearnaley, EJ and Hansel, CEM. The risk taken in crossing a road. Journal of the Operational Research Society, Vol. 6, 1955, pp. 120-128.

(31) Brewer, AM, Fitzpatrick, K, Whitacre, AJ and Lord, D. Exploration of pedestrian gap-acceptance behaviour at selected locations. Transportation Research Record, Vol. 1982, 2006, pp. 132-140.

(32) Yannis, G, Golias, J and Papadimitriou, E. Modeling crossing behavior and accident risk of pedestrians. ASCE Journal of Transportation Engineering, Vol. 133, 2007, pp. 634-644.

(33) Yannis, G, Papadimitriou, E and Theofilatos, A. Pedestrian gap acceptance for mid-block street crossing. Transportation Planning and Technology, Vol. 36, 2013, pp. 450-462.

(34) Das, S, Mansk, CF and Manuszak, MD. Walk or wait? An empirical analysis of street crossing decisions. Journal of Applied Economics, Vol. 20, 2005, pp. 529-548.

(35) Tiwari, G, Bangdiwala, S, Saraswat, A and Gaurav, S. Survival analysis: pedestrian risk exposure at signalized intersections. Transportation Research Part F: Traffic Psychology and Behaviour, Vol. 10, 2007, pp. 77-89.

(36) Clarkson J, Thorpe N, Goodman P, Bell MC and Galatioto F. Modelling the interactions between pedestrians and vehicles in shared spaces. $46^{\text {th }}$ Annual Conference of the Universities Transport Study Group (UTSG) Newcastle upon Tyne, UK, 2014.

(37) Washington, SP, Karlaftis, MG and Mannering, FL. Statistical and econometric methods for transportation data analysis. Chapman \& Hall/CRC, USA, 2010. 


\section{LIST OF TABLES AND FIGURES}

TABLE 1 Key gap acceptance behaviour variables in the Exhibition Road site before and after redevelopment

TABLE 2 Results of binary logistic regression for gap acceptance at the three locations of Exhibition Road

FIGURE 1 Exhibition Road before (left) and after redevelopment (right)

FIGURE 2 (a) Location and general alignment of the monitored pedestrian crossing occurrences at the Exhibition Road site; (b) camera locations in the before- (left) and aftermonitoring (right)

FIGURE 3 Critical gap calculation at the three locations of the Exhibition Road site 
TABLE 1: Key gap acceptance behaviour variables in the Exhibition Road site before and after redevelopment

\begin{tabular}{|c|c|c|c|c|c|c|}
\hline \multirow{2}{*}{ (a) } & \multicolumn{2}{|c|}{ L1 } & \multicolumn{2}{|c|}{$\mathbf{L 2}$} & \multicolumn{2}{|c|}{$\mathbf{L 3}$} \\
\hline & Before & After & Before & After & Before & After \\
\hline Number of observed gaps & 172 & 132 & 268 & 189 & 172 & - \\
\hline \multicolumn{7}{|l|}{ Gap length (seconds) } \\
\hline Mean & 3.775 & 3.588 & 3.510 & 3.280 & 3.535 & - \\
\hline Standard deviation & 1.711 & 1.330 & 1.403 & 1.536 & 1.475 & - \\
\hline Coefficient of variation & 0.453 & 0.371 & 0.400 & 0.468 & 0.417 & - \\
\hline \multicolumn{7}{|l|}{ Vehicle speed $(\mathrm{km} / \mathrm{h})$} \\
\hline Mean & 40.480 & 28.971 & 42.671 & 41.259 & 42.217 & - \\
\hline Standard deviation & 4.386 & 2.767 & 3.447 & 3.472 & 3.974 & - \\
\hline Coefficient of variation & 0.108 & 0.096 & 0.081 & 0.084 & 0.094 & - \\
\hline \multirow{2}{*}{ (b) } & \multicolumn{2}{|c|}{ L1 } & \multicolumn{2}{|c|}{$\mathbf{L 2}$} & \multicolumn{2}{|c|}{$\mathbf{L 3}$} \\
\hline & Before & After & Before & After & Before & After \\
\hline Number of accepted gaps & 44 & 33 & 49 & 42 & 42 & - \\
\hline \multicolumn{7}{|l|}{ Waiting time (seconds) } \\
\hline Mean & 9.245 & 9.516 & 13.737 & 9.275 & 9.167 & - \\
\hline Standard deviation & 5.209 & 5.094 & 4.383 & 2.448 & 2.725 & - \\
\hline Coefficient of variation & 0.564 & 0.535 & 0.319 & 0.264 & 0.297 & - \\
\hline \multicolumn{7}{|l|}{ Crossing time (seconds) } \\
\hline Mean & 5.124 & 5.665 & 5.223 & 5.646 & 5.028 & - \\
\hline Standard deviation & 1.112 & 0.816 & 0.695 & 0.671 & 0.809 & - \\
\hline Coefficient of variation & 0.217 & 0.144 & 0.133 & 0.119 & 0.161 & - \\
\hline \multicolumn{7}{|l|}{ Crossing speed $(\mathrm{km} / \mathrm{h})$} \\
\hline Mean & 2.972 & 2.599 & 2.810 & 2.587 & 2.937 & - \\
\hline Standard deviation & 0.798 & 0.418 & 0.415 & 0.319 & 0.478 & - \\
\hline Coefficient of variation & 0.268 & 0.161 & 0.148 & 0.123 & 0.163 & - \\
\hline
\end{tabular}


TABLE 2: Results of binary logistic regression for gap acceptance at the three locations of Exhibition Road

\begin{tabular}{|c|c|c|c|c|c|c|c|}
\hline \multicolumn{4}{|c|}{ Exhibition Road main body (L1) - Before } & \multicolumn{4}{|c|}{ Exhibition Road main body (L1) - After } \\
\hline Attribute & Coeff. (B) & Std. Err. & Sig. & Attribute & Coeff. (B) & Std. Err. & Sig. \\
\hline Group & -1.417 & 0.897 & .114 & Group & -0.383 & 0.656 & .559 \\
\hline Elderly & -0.746 & 0.678 & .271 & Elderly & -1.367 & 0.702 & .052 \\
\hline Children & -0.022 & 1.088 & .984 & Children & 1.053 & 1.440 & .465 \\
\hline Long gap & 4.684 & 0.752 & .000 & Long gap & 3.702 & 0.661 & .000 \\
\hline Fast vehicle & -2.025 & 0.954 & .034 & Fast vehicle & -2.655 & 1.606 & .098 \\
\hline Constant & -1.565 & 0.349 & .000 & Constant & -1.551 & 0.398 & .000 \\
\hline \multicolumn{4}{|c|}{$\begin{array}{l}\text { Number of observations }=172 ; \text { chi }^{2}=92.077 ; \text { Sig. }=.000 \\
\text { Pseudo- } R^{2}(\text { Nagelkerke })=0.610\end{array}$} & \multicolumn{4}{|c|}{$\begin{array}{l}\text { Number of observations }=132 ; \text { chi }^{2}=51.641 ; \mathrm{Sig} .=.000 \\
\text { Pseudo- } R^{2}(\text { Nagelkerke })=0.479\end{array}$} \\
\hline \multicolumn{4}{|c|}{ Cromwell Road junction (L2) - Before } & \multicolumn{4}{|c|}{ Cromwell Road junction (L2) - After } \\
\hline Attribute & Coeff. (B) & Std. Err. & Sig. & Attribute & Coeff. (B) & Std. Err. & Sig. \\
\hline Group & -2.530 & 1.089 & .020 & Group & -1.032 & 0.778 & .184 \\
\hline Elderly & -1.508 & 0.768 & .050 & Elderly & -1.259 & 0.732 & .086 \\
\hline Children & -1.536 & 0.959 & .109 & Children & -0.675 & 0.913 & .460 \\
\hline Long gap & 7.241 & 1.192 & .000 & Long gap & 5.436 & 0.781 & .000 \\
\hline Fast vehicle & -5.069 & 1.599 & .002 & Fast vehicle & -2.689 & 1.126 & .017 \\
\hline Constant & -2.119 & 0.506 & .000 & Constant & -1.976 & 0.602 & .001 \\
\hline \multicolumn{4}{|c|}{$\begin{array}{l}\text { Number of observations }=268 ; \text { chi }^{2}=181.104 ; \text { Sig. }=.000 \\
\text { Pseudo- } R^{2}(\text { Nagelkerke })=0.800\end{array}$} & \multicolumn{4}{|c|}{$\begin{array}{l}\text { Number of observations }=189 ; \text { chi }^{2}=126.221 ; \text { Sig. }=.000 \\
\text { Pseudo- } R^{2}(\text { Nagelkerke })=0.746\end{array}$} \\
\hline \multicolumn{4}{|c|}{ Thurloe Street (L3) - Before } & & & & \\
\hline Attribute & Coeff. (B) & Std. Err. & Sig. & & & & \\
\hline Group & -0.409 & 0.481 & .395 & & & & \\
\hline Elderly & -2.066 & 0.728 & .005 & & & & \\
\hline Children & -1.582 & 0.802 & .049 & & & & \\
\hline Long gap & 4.025 & 0.724 & .000 & & & & \\
\hline Fast vehicle & \multicolumn{3}{|c|}{ dropped due to collinearity } & & & & \\
\hline Constant & -1.339 & 0.337 & .000 & & & & \\
\hline \multicolumn{4}{|c|}{$\begin{array}{l}\text { Number of observations }=172 ; \mathrm{chi}^{2}=59.808 ; \mathrm{Sig} .=.000 ; \\
\text { Pseudo }-R^{2} \text { (Nagelkerke) }=0.438\end{array}$} & & & & \\
\hline
\end{tabular}


(a)
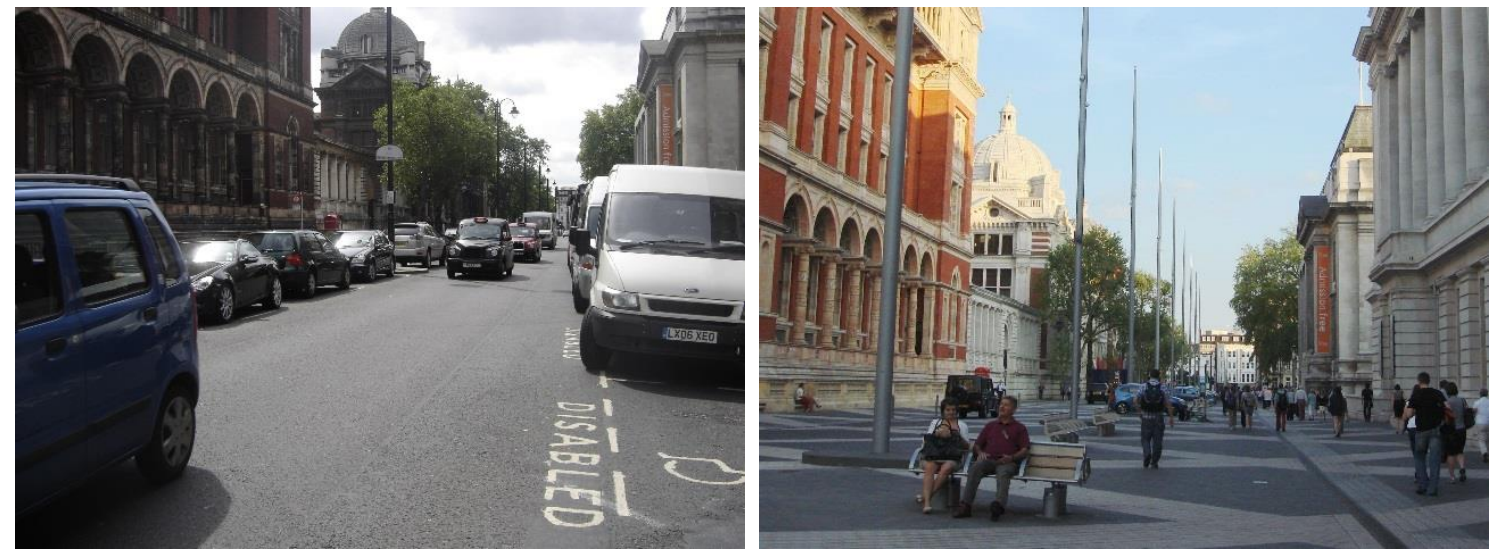

(b)

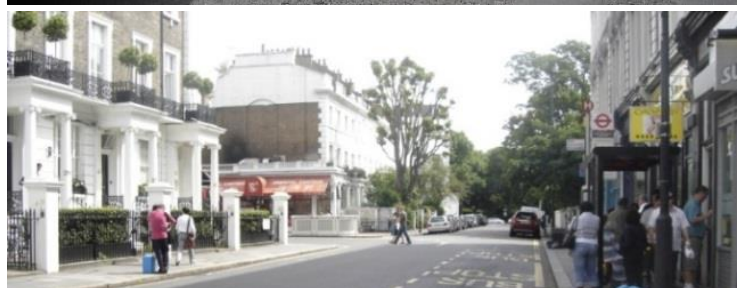

(c)
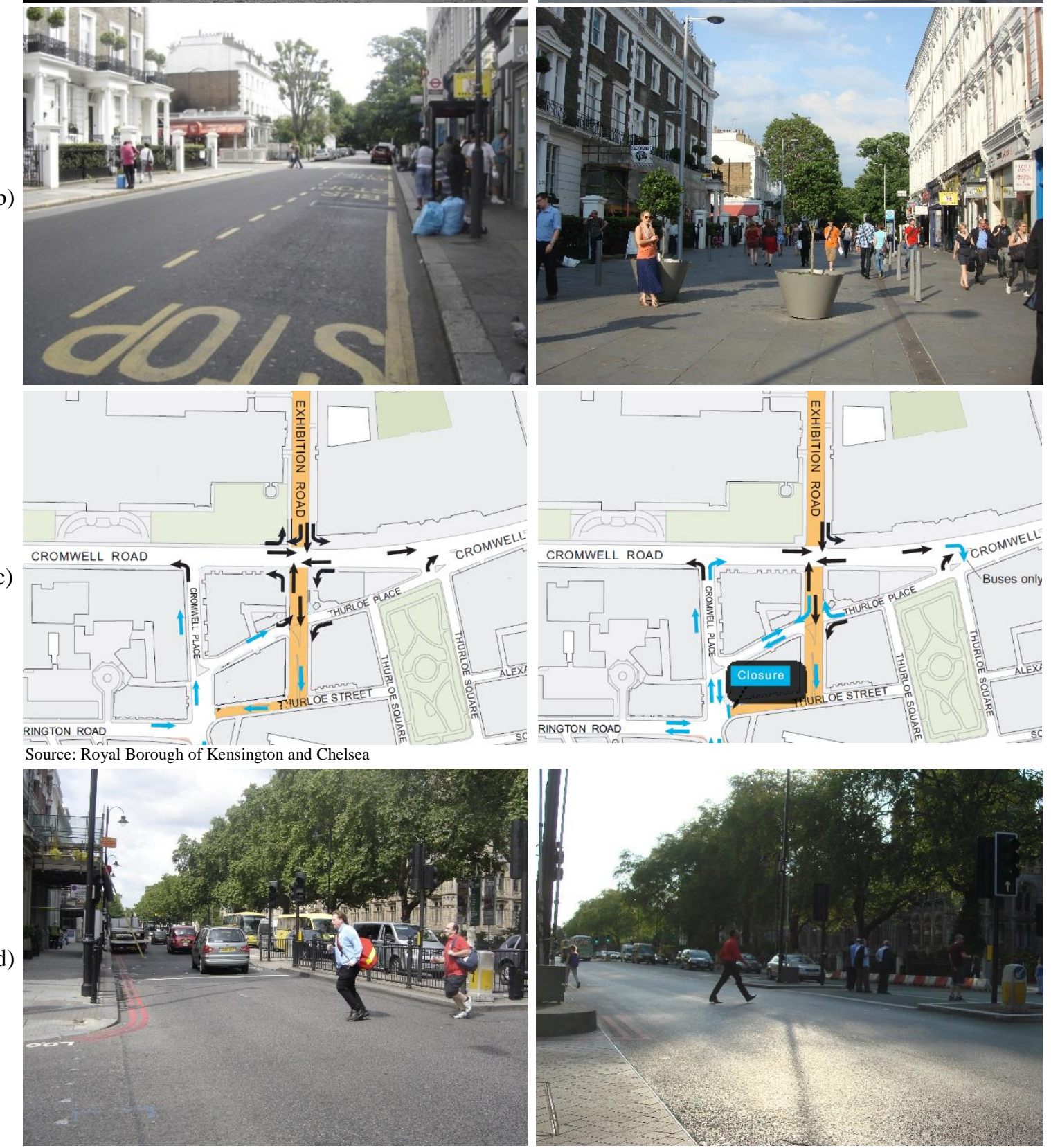

FIGURE 1: Exhibition Road before (left) and after redevelopment (right) 
(a)

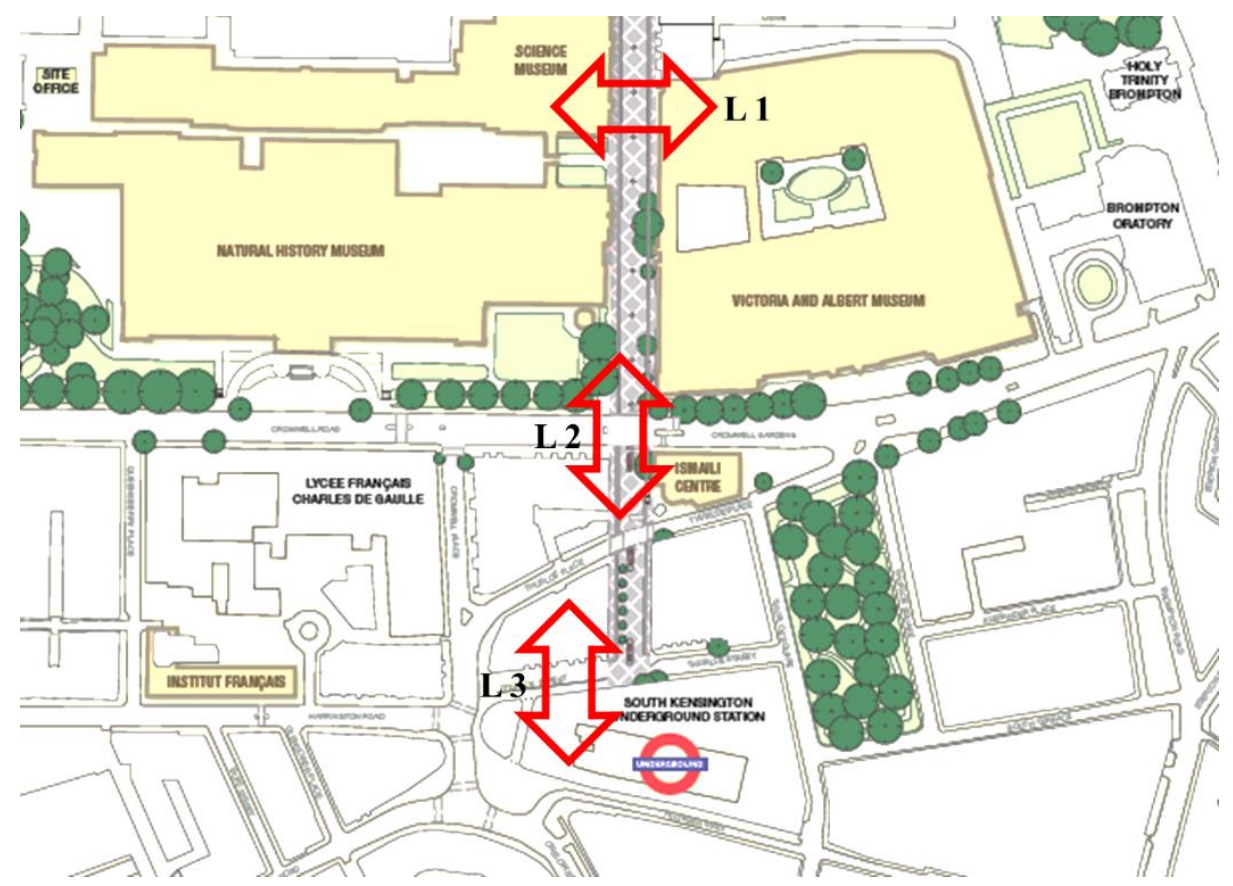

(b)
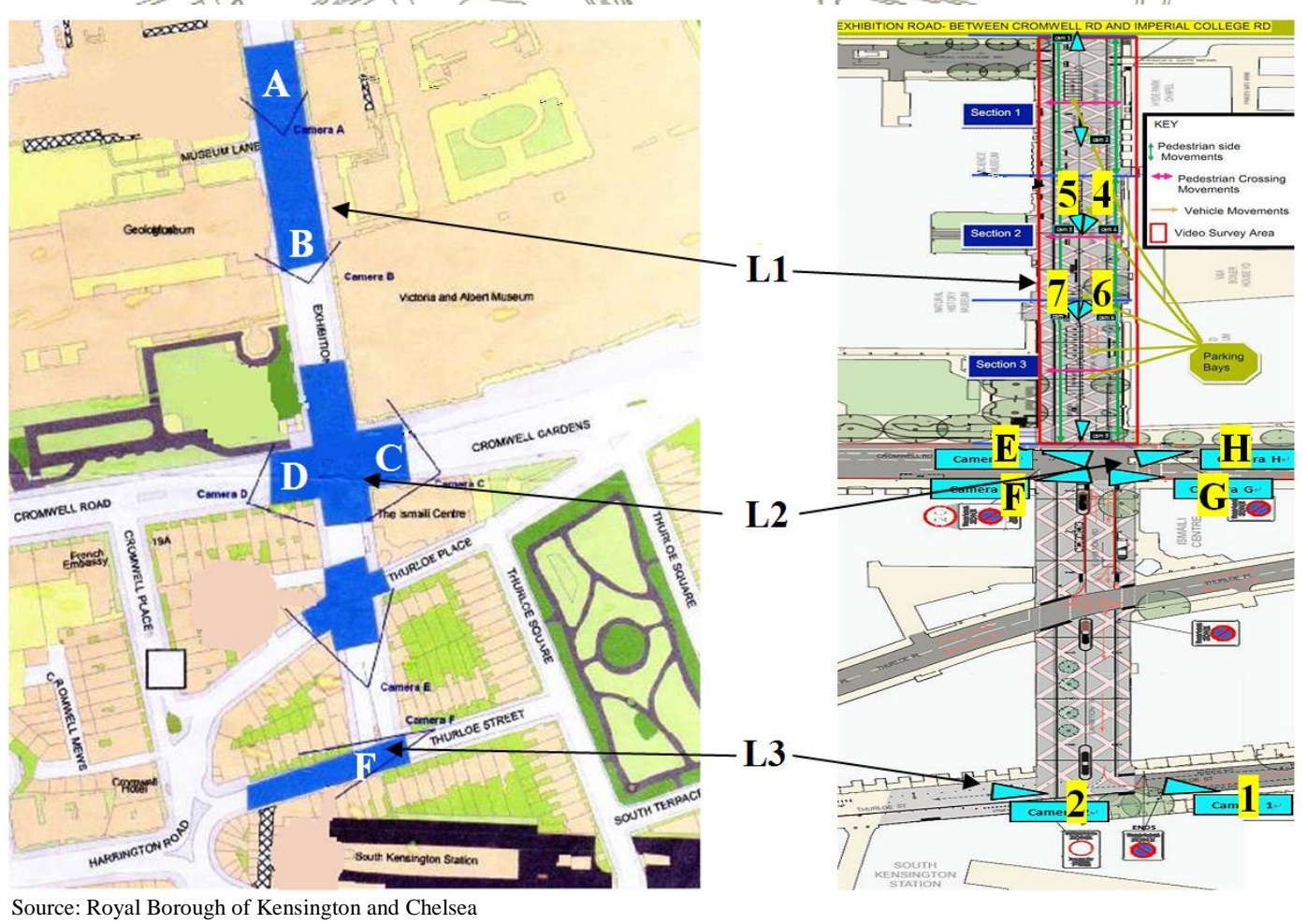

FIGURE 2: (a) Location and general alignment of the monitored pedestrian crossing occurrences at the Exhibition Road site; (b) camera locations in the before- (left) and after-monitoring (right) 


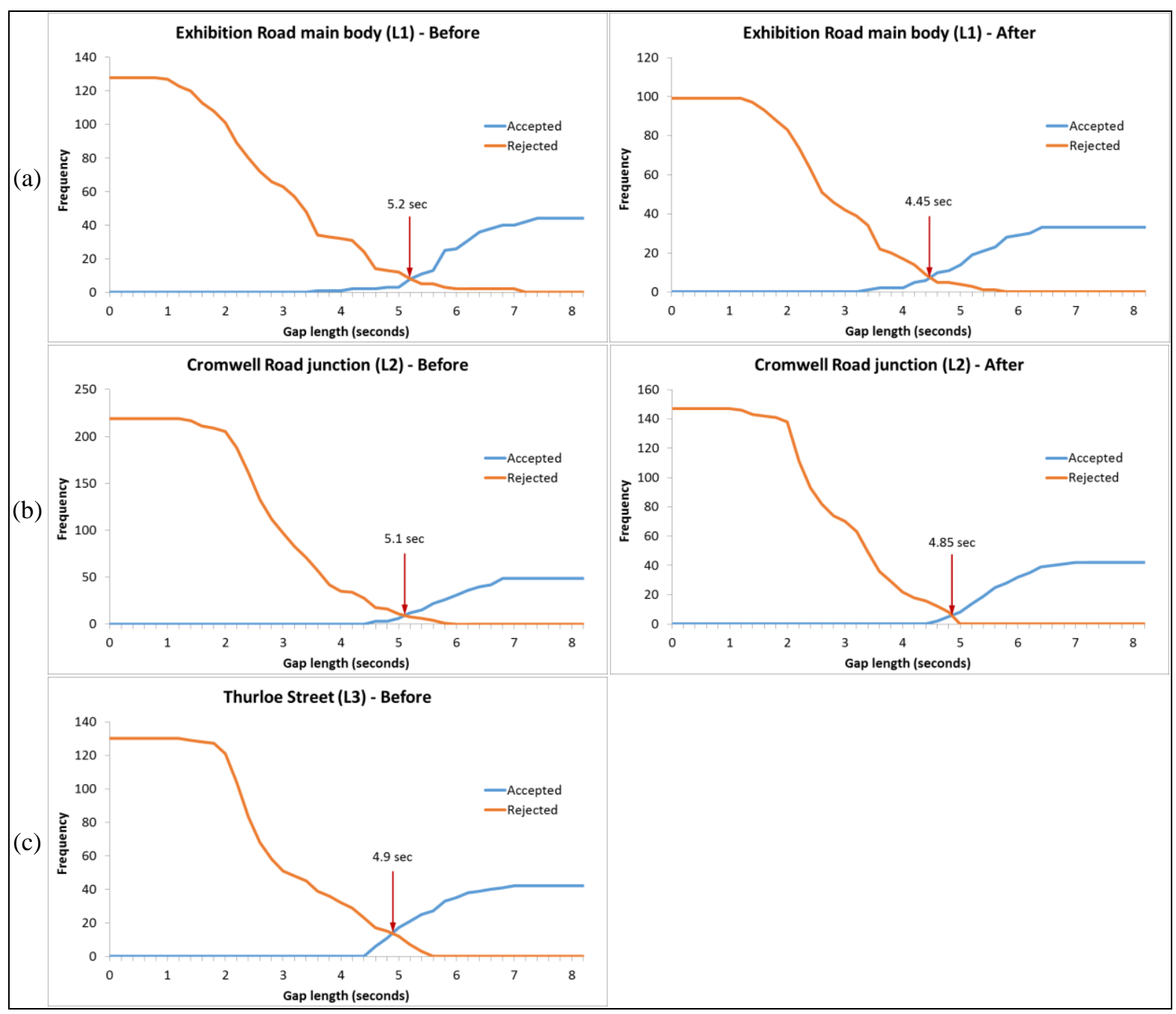

FIGURE 3: Critical gap calculation at the three locations of the Exhibition Road site 